\section{Color-coded methodology for deformable mirrors}

\author{
Paola Sansoni, Daniela Fontani, Franco Francini, \\ Luca Mercatelli, and David Jafrancesco \\ CNR - Istituto Nazionale di Ottica Applicata, \\ Largo E. Fermi 6, Firenze 50125, Italy \\ E-mail: paola.sansoni@inoa.it
}

\begin{abstract}
We describe an experimental procedure for the reconstruction of the geometrical parameters of a reflecting surface. The method is based on the projection of a luminous pattern constituted by colored points. The successive chromatic selection reduces the complexity of the acquired image. We show that the colors of the points comprising the pattern are modified by the combined effect of the CCD camera and the projection system. In some situations, the chromatic components of the pattern points can generate ambiguity. Consequently, the number of colors to be projected and their chromatic separation must be studied to enable the simultaneous projection of an elevated number of points and colors. () 2006 Society of Photo-Optical Instrumentation Engineers.
\end{abstract} [DOI: 10.1117/1.2333452]

Subject terms: optical testing; color; light; projection systems.

Paper 060115LR received Feb. 22, 2006; revised manuscript received May 25, 2006; accepted for publication May 30, 2006; published online Aug. 23, 2006.

\section{Forced Curvature Mirrors for Heliostats}

Some types of heliostats working in solar plants use concave mirrors to concentrate sunlight on an absorber. The mirrors used for this purpose are originally plane and their curvature is artificially induced. Typically, the curvature radius of these large mirrors exceeds $20 \mathrm{~m}$.

The mirror under test is a hexagonal reflecting surface, which is inscribed within a 1-m-diam circle. The reflecting surface leans on a metallic $83-\mathrm{cm}$-diam ring, which has a mechanism that creates traction on the central point of the mirror by means of a screw. This traction screw is fixed to a circular $15-\mathrm{cm}$-diam plate, which is glued to the rear part of the mirror. The curvature of the mirror can be modified by rotating the traction screw. The described technique was developed to perform noncontact measurements on largesize mirrors. When it is applied to the 1-m heliostat, it enables us to associate a traction value (number of turns of the traction screw) to the morphological characteristics of the heliostat surface.

\section{Measurement Principle}

The scheme of the measurement system illustrated in Fig. 1 is composed of an LCD projector, which is used to indirectly reproduce on a screen a pattern constituted by an array of colored points. The rays, before arriving at the screen, are reflected by the surface $M$, which is under test. Considering the path of a ray reflected by a plane or a curve surface of the mirror, whose curvature is induced by a deformation, $S_{1}$ (for a plane mirror) and $S_{2}$ (for a curved mirror) represent the nodal points of the optical projection system, as shown in Fig. 2. The colored points constituting

0091-3286/2006/\$22.00 @ 2006 SPIE the pattern are captured by means of a digital camera, which acquires a couple of images, before and after the mirror deformation; $I_{M}$ is the image of the deformed mirror and $I_{R}$ is the reference image of the plane mirror.

The geometrical features of the surface are determined by elaborating the values of the local slope, which are obtained using an algorithm that takes into account the position of the corresponding points in the images $I_{M}$ and $I_{R}$.

The deviation of the luminous ray depends on the local slope of the $M$ surface at the point $P$, where the reflection takes place, as illustrated in Fig. 2. If the surface is plane the incidence angle is $\alpha$; whereas when the surface is curve, considering that its local slope is $\Delta \varphi$, the corresponding incidence angle becomes $\alpha-\Delta \varphi$. Here $S_{1}$ and $S_{2}$ indicate the position of a ray reflected by the point $P$, which has as vertical coordinate $y$. In practice, this value represents the distance of a colored pattern point from the origin, which can be set at the base of the mirror. The ray reflected by the plane mirror arrives on the screen at $S_{1}$ and $S_{2}$ is the corresponding displaced point on flexing the mirror.

$O S_{1}=y+D \tan \alpha$

$O S_{2}=y+D \tan (\alpha-\Delta \varphi)$,

where $D$ is the distance between screen and mirror, $\alpha$ $=\arctan (y / L)$, and $L$ is the distance between source and mirror. After some calculations, setting $C=\left(O S_{1}-O S_{2}\right) / D$ results in

$\tan \Delta \varphi=\frac{-C}{C \tan \alpha-\tan ^{2} \alpha-1}$

Applying Eq. (1) for all the corresponding points within the two images $I_{R}$ and $I_{M}$, the values of the slope can be obtained for the whole surface. In this procedure, we assumed that the point $P$ is the same for both rays, striking points $P$ and $S_{1}$ (for the plane mirror) or striking points $P$ and $S_{2}$ for the reflection on the flexed mirror. This approximation causes an error in the calculation of the $S_{2}$ coordinates less than $0.005 \%$ for a curvature radius of the order of $20 \mathrm{~m}$ (from Fig. 5 in Sec. 4, the curvature examined exceeds $60 \mathrm{~m})$.

The variations in the distance $L$ were considered in the software developed to provide the reconstruction data. This software elaborates the two dimensions in the plane perpendicular to the optical axis separately, considering each coordinate $x$ and $y$.

\section{Experimental Measurement of the Local Slope Using Colored Patterns}

This technique represents the evolution of the method currently used in our laboratories, which is based on the projection of monochromatic gratings. ${ }^{1-3}$ When LCD projectors became reasonably priced and widely diffused, the related techniques for projecting color pattern on diffusing objects $^{4-11}$ obtained development and dissemination. The aim of this work is to experiment with the use of these techniques to detect the morphological characteristics of mirrors.

The experimental procedure employs a cluster of colored luminous beams that are projected on the test mirror 


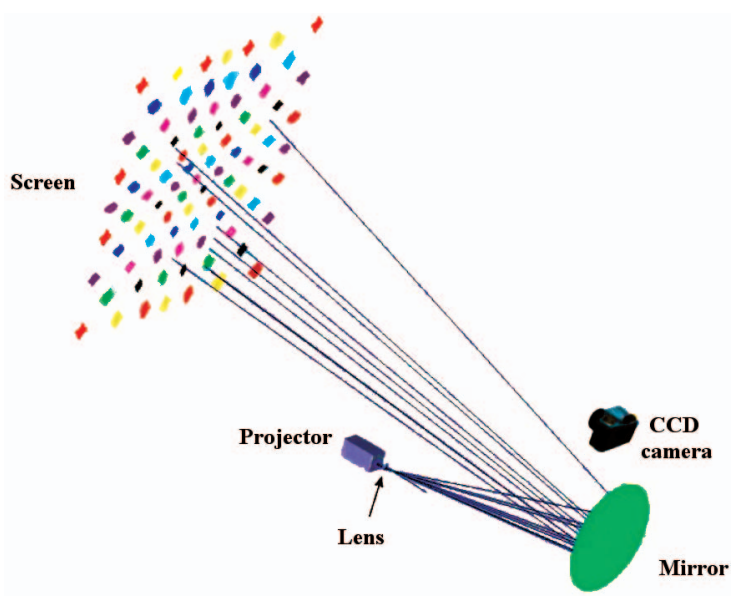

Fig. 1 Experimental setup for colored pattern projection and CCD image acquisition.

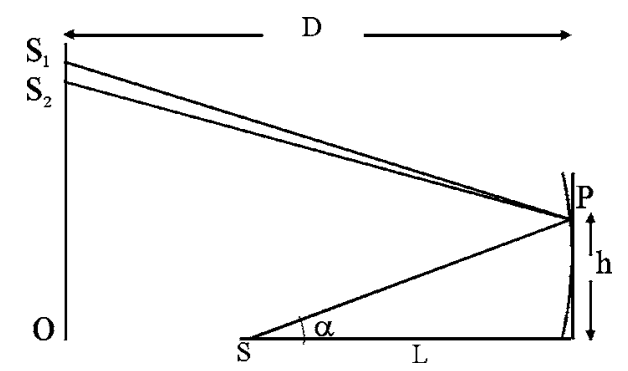

Fig. 2 Displacement between the rays reflected from the plane mirror and the curved mirror.

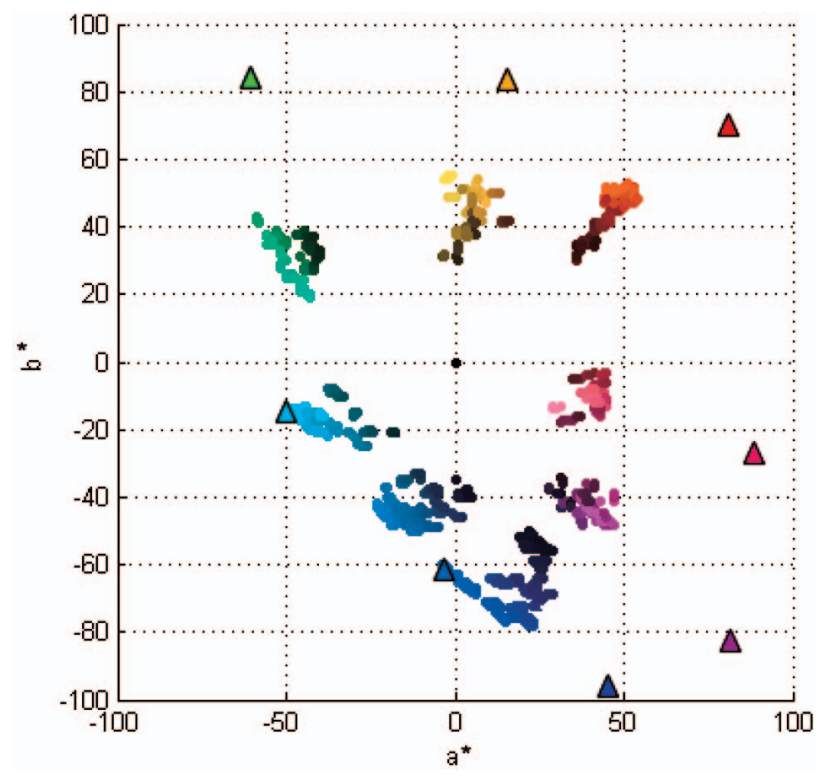

Fig. 3 Photographed colors with respect to the projected ones in the CIELAB diagram.

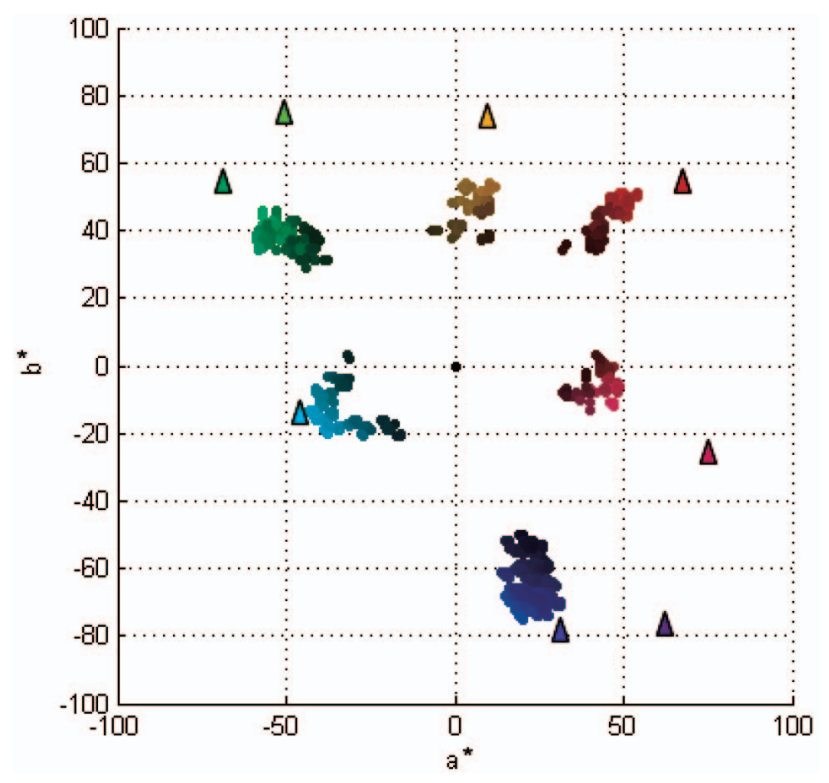

Fig. 4 Photographed and projected colors with equal spacing in the CIELAB diagram.

by means of a LCD projector (Philips LC4341). The deformed image and the reference one are acquired by the CCD photographic camera (FUJIFILM FinePix Slpro, $3040 \times 2016$ max). Typical values of the setup parameters are total $\mathrm{CCD}$ size $=23.3 \times 15.6 \mathrm{~mm}, L \sim 3 \mathrm{~m}, D \sim 3 \mathrm{~m}$, spot number $=50,000$ to 100,000 , and spot size $\sim 3$ $\times 3 \mathrm{~mm}$. The data of the mirror profile were calculated with an error of about $0.02 \mathrm{~mm}$ in the $z$ coordinate. The precision of our method is not comparable with other techniques, such as interferometeric measurements reaching much higher resolution. Nevertheless, the use of an interferometer to measure such large mirrors requires a sizable setup.

The introduction of a chromatic code in the pattern simplifies the algorithm used to reconstruct the slope since it reduces the ambiguity in the search of the corresponding points within the deformed and reference surfaces.

By applying suitable procedures of color filtering, it is possible to decrease the complexity of the image of a factor equal to the number of the colors employed. The superim-

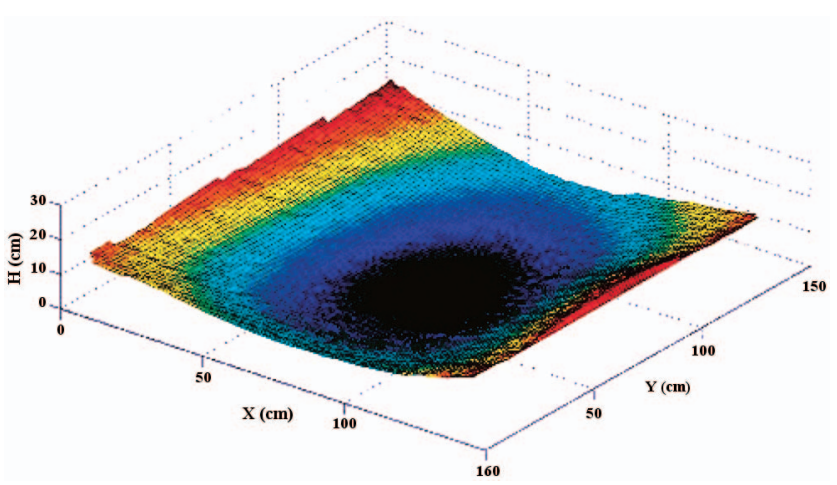

Fig. 5 A 3-D reconstruction of the mirror profile. 
position of the $I_{M}$ and $I_{R}$ images shows the displacement of the corresponding points, which are unambiguously indicated by the same color.

\section{Selections of the Pattern Colors}

The images projected on the screen are composed of $N$ matrices of points pertaining to a defined sequence. The points of each matrix must have the same color to easily separate the matrices by applying a software filter.

The chromatic codification was introduced to facilitate this operation of matrix separation, and the choice of the color set is a crucial element. The selection of the eight colors was carried out referring to the hue values and optimizing their chromatic separation, which was studied in the CIELAB color space $L^{*} a^{*} b^{*}$ (Ref. 12). The chromatic separation between projected and photographed patterns is visualized in Figs. 3 and 4 , reporting the $a^{*}$ and $b^{*}$ coordinates.

The selection of the number of matrices, which was limited to eight, derives from experimental tests with our CCD camera and projector system, which are the main elements altering the color pattern. It was verified that, with our optical setup fixing diaphragm and the integrating time of the camera, this is the maximum number of colors that can be separated in the photographed image. The final result of these chromatic tests is presented in Fig. 3, which reports the CIELAB coordinates corresponding to the colored pattern. In Fig. 3, the photographed pattern is compared to the original pattern, which is reported as triangles. Note that the eight colors appear correctly separated in the photographed image, as they were in the original pattern. The combined effect of CCD camera and LCD projector modifies the colors and every point becomes a large spot.

It is interesting to compare Fig. 3 with Fig. 4, which represents an intermediate phase of these chromatic tests, in which the eight colors were selected with equal spacing in the hue value. Figure 4 is analogous to Fig. 3, representing in the CIELAB plane the colored pattern before and after acquisition. In Fig. 4 the original pattern, where the eight colors appear as points, is indicated with triangles. The other CIELAB coordinates correspond to the photographed image, where there are only six distinct colors.

It can be concluded that, even though there are some color variations due to the combination of LCD projector and digital camera, the eight matrices are still distinguishable if the colors of the points are accurately selected.

Figure 5 represents the $3-\mathrm{D}$ reconstruction of the surface of the heliostat with a traction corresponding to four screw turns. The mirror deformations are very weak: the sagitta in the center turns out to be of the order of few millimeters. The corresponding curvature radius of the 1 - $\mathrm{m}$ mirror results in $40 \mathrm{~m}$ for a sagitta of $3 \mathrm{~mm}$ and $60 \mathrm{~m}$ for a sagitta of $2 \mathrm{~mm}$.

\section{Conclusions}

Noncontact shape assessment performed on huge dimension objects has always presented practical problems caused simply by the dimensions of the related measurement equipment. While the shape assessment on diffusing surfaces can be performed by employing image analysis techniques or projecting laser lines or gratings, if the examined surface is a mirror, with a significant reflection coefficient, it is not convenient to obtain the image of the sample. It is preferable to use procedures that consider the object an integrant part of the optical measurement system. The technique illustrated in this paper foresees the use of a cluster of luminous beams, with colors based on a code that facilitates the identification of the rays pertaining to the various points of the sample. The measurement setup includes a LCD projector and a digital camera. The described methodology was applied to assess the profile on an heliostat with $1 \mathrm{~m}$ of external dimension and curvature radius exceeding $20 \mathrm{~m}$. The method was adapted to the optical setup. Since the projector and the digital camera induce modifications in the pattern colors, the choice of the color set requires a specific study. These chromatic coded techniques are simple, fast, and versatile, and they can be applied to other scientific or industrial measurements, controls, and tests.

\section{References}

1. D. Malacara, Optical Shop Testing, Wiley Series in Pure and Applied Optics, J. Wiley and Sons, New York (1978).

2. L. Mercatelli, D. Fontani, F. Francini, D. Jafrancesco, and P. Sansoni, "Shape detection by 'Reflection Moiré Method' with optical design validation," in Optical Metrology - SPIE Europe 5856-28, 377-384 (2005).

3. L. Salas, E. Luna, J. Salinas, and V. Garcia, "Profilometry by fringe projection," Opt. Eng. 42(11), 3307-3314 (2003).

4. Z. J. Geng, "Rainbow three-dimensional camera: new concept of high-speed three-dimensional vision systems," Opt. Eng. 35(2), 376-383 (1996)

5. C.-H. Hu and Y.-W. Qin, "Digital color encoding and its application to the moiré technique," Appl. Opt. 38(16), 3682-3685 (1997).

6. P. S. Huang, Q. Hu, F. Jin, and F.-P. Chiang, "Color-encoded digital fringe projection technique for high-speed three-dimensional surface contouring," Opt. Eng. 38(6), 1065-1071 (1999).

7. W. Liu, Z. Wang, G. Mu, and Z. Fang, "Color-coded projection grating method for shape measurement with a single exposure," Appl. Opt. 39(20), 3504-3508(2000).

8. M.-S. Jeong and S.-W. Kim, "Color grating projection moirè with time-integral fringe capturing for high-speed 3-D imaging," Opt. Eng. 41(8), 1912-1917 (2002).

9. P. S. Huang, C. Zhang, and F.-P. Chiang, "High-speed 3-D shape measurement based on digital fringe projection," Opt. Eng. 42(1), 163-168 (2003).

10. J. Pan, P. S. Huang, and F.-P. Chiang, "Color-coded binary fringe projection technique for 3-D shape measurement," Opt. Eng. 44(2), 023606 (2005).

11. J. Pan, P. S. Huang, and F.-P. Chiang, "Color phase-shifting technique for three-dimensional shape measurement," Opt. Eng. 45(1), 013602 (2006).

12. Commission International de l'éclairage, CIE Publ. N. 17.4, Vocabulaire international de l'éclairage 1987 publication 50(845) (1987). 